\section{Imaging and microscopy}

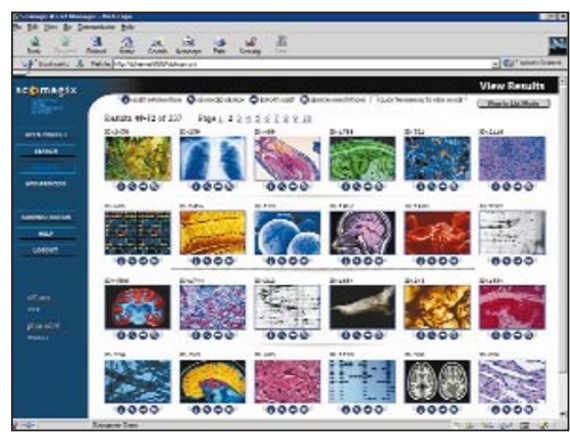

\section{Image informatics}

Scimagix's (Redwood Shores, CA) Scientific Image Management System (SIMS) is designed to store, browse, and search images generated in drug discovery $\mathrm{R} \& \mathrm{D}$. Images can be viewed via a Web browser as collections or as the result of a search for images with specific characteristics, and can be integrated with other experimental data. The software is Web/Oracle-based, and optional modules are available for additional analysis and mining.

http://www.scimagix.com

RIN: 1247

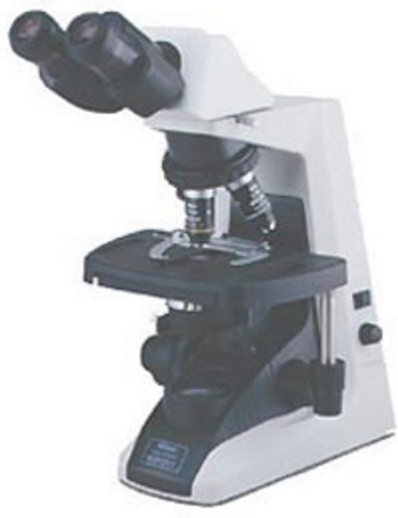

\section{High-quality optics}

From Nikon (Tokyo, Japan) the Eclipse E200 educational microscope incorporates the company's renowned $\mathrm{CFI}_{60}$ infinity optical system, resulting in stunningly sharp and clear images at any magnification, and at an affordable price. Improved ergonomic design allows for greater user comfort, and Nikon's unique refocusing stage eliminates the need to refocus after loading or unloading a slide, thereby making specimen handling safer and easier. The Eclipse E200 is compatible with many accessories for higher grade Eclipse series microscopes.

http://www.nikon.com

RIN: 1248

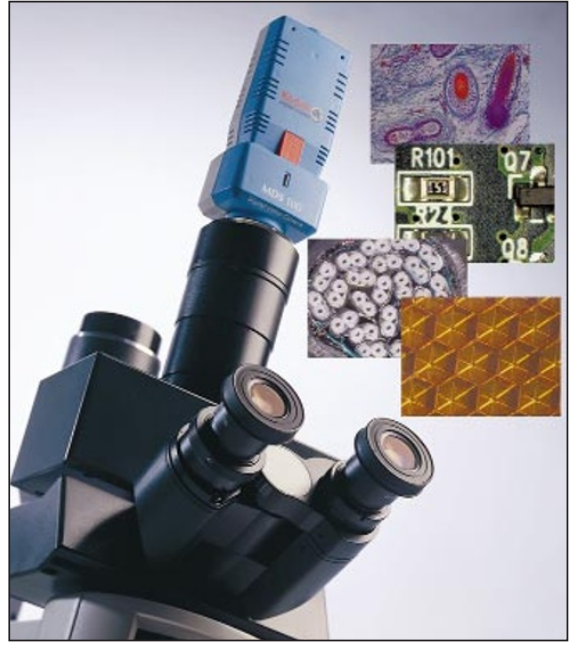

\section{Digital documentation}

Kodak's (New Haven, CT) Microscopy Documentation System (MDS) 100 provides an affordable imaging solution for a variety of laboratory applications. The included software allows users to preview, capture, annotate, edit, and save high-resolution still images, video streams, and time-lapse sequences, both on and off a microscope. The system works equally well under most microscopy illumination conditions, including bright-field, dark-field, polarized, phase contrast, and Nomarski illumination.

http://www.kodak.com

RIN: 1249

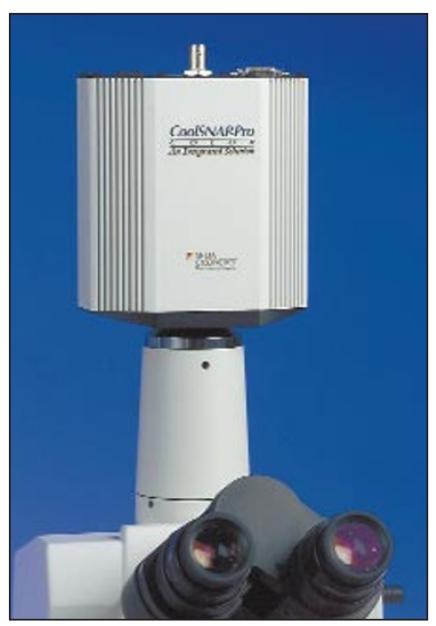

\section{Imaging kit}

The CoolSNAP-Pro Digital Kit from Media Cybernetics (Silver Spring, ND) offers users advanced imaging technology for microscopy and inspection applications. The kit includes software, a 36-bit color digital or 12-bit monochrome digital camera, and a PCI interface card and cable, and features include easy installation and set-up, built-in default and user-definable camera set-up files, automatic white balance correction and dark-field subtraction, fast image and color preview, and advanced image capture options.

http://www.mediacy.com

RIN: 1250

\section{Thermocycling}

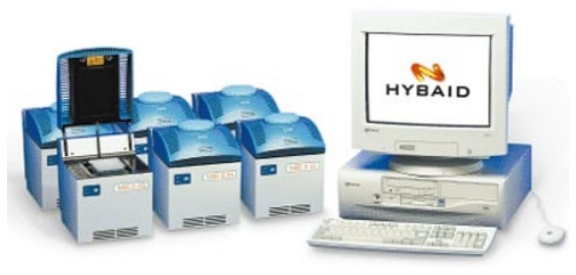

\section{PCR network}

The MultiBlock System (MBS) from Hybaid (Ashford, UK) consists of a central computer and software that can control up to 30 individual blocks. Different block formats are available, including $0.2 \mathrm{ml}, 0.5 \mathrm{ml}, 384$ and gradient blocks. The choice of the number and type of each block can be specified, giving customers complete flexibility and the ability to customize their own PCR system. The software features a large graphical user interface, easy printout of experimental data, at-a-glance block status report, and real-time block data.

http://www.hybaid.co.uk RIN: 1251

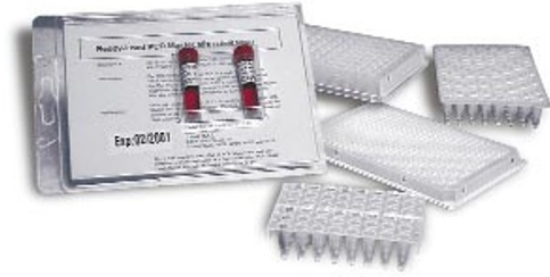

\section{Instant PCR}

ABGene's (Epsom, UK) range of ReddyMix PCR Master Mixes contain all the components for PCR except template and primers. Uniquely formulated with a red dye and precipitant for direct loading of PCR products onto agarose gels for electrophoresis, ReddyMix Master Mix dramatically reduces both PCR set-up time and post-PCR analysis time. The red indicator dye has no negative effect on PCR performance, and can even enhance the efficiency of PCR reactions. It is available in six $\mathrm{MgCl}_{2}$ concentrations, in single-tube format or pre-aliquoted into reaction tubes or plates for maximum efficiency. http://www.abgene.com

RIN: 1252 